\title{
Dor Crônica sob a Ótica Comportamental: Compreensão e Possibilidades de Intervenção
}

\author{
Chronic Pain under Behavioral Optics: Understanding and Intervention Possibilities
}

Dolor Crónica bajo la Óptica Comportamental: Comprensión y Posibilidades de Intervención

\author{
Cristiane Rodrigues Lopes ${ }^{1}$ \\ Vanessa Ferrari \\ Cynthia Carvalho Jorge \\ Universidade Paranaense (UNIPAR)
}

\begin{abstract}
Resumo
A média da população brasileira que se queixa ou sofre de dor crônica é ampla e tem-se caracterizado em um grande desafio para as ciências da saúde. Portanto o objetivo deste trabalho foi realizar uma análise conceitual a respeito da compreensão da dor crônica sob a perspectiva comportamental, abordando técnicas de intervenção psicológicas utilizadas para o manejo da dor. Para tanto, foi realizada uma busca na literatura por meio de artigos e livros, que relatam a aplicabilidade das teorias comportamentais no tratamento da dor crônica e alguns estudos científicos que corroboram a eficácia dessa teoria para o manejo da dor. A partir da literatura encontrada, conclui-se que as teorias comportamentais contribuem para o entendimento e tratamento da dor crônica, tendo em vista que contingências ambientais podem ter um papel de destaque no início, na gravidade e na manutenção da dor. Acredita-se, portanto, que esses achados possam ser de grande valia para os profissionais contribuindo com melhores práticas no âmbito da saúde.
\end{abstract}

Palavras-chave: dor crônica, comportamental, terapia comportamental, manejo da dor

\begin{abstract}
The average Brazilian population which suffers or complaints about chronic pain is wide and is a major challenge to medicine and psychology. The purpose of this work is to perform a conceptual analysis on the understanding of the chronic pain from a behavioral perspective, addressing techniques of psychological intervention used for pain management. Therefore, a literature search was executed through articles and books, which relate the applicability of behavioral theories in the treatment of chronic pain, and some scientific studies that corroborate for the effectiveness of this theory for pain management. From the literature found, we conclude that behavioral norms contribute to the understanding of chronic pain, regarding environmental problems and thoughts may play an important role in the origin, severity, and management of pain. We, therefore, believe that these conclusions may be of great utility for professionals, contributing to best practices in the health scope.
\end{abstract}

Keywords: chronic pain, behavioral, behavioral therapy, pain management

\section{Resumen}

La media de la población brasileña que se queja o sufre de dolor crónico es amplia y se ha caracterizado en un gran desafío a la medicina ya la psicología. Por lo tanto el objetivo de este trabajo fue realizar un análisis conceptual acerca de la comprensión del dolor crónico bajo la perspectiva comportamental, abordando técnicas de intervención psicológicas utilizadas para el manejo del dolor. Para ello se realizó una búsqueda en la literatura por medio de artículos y libros, que relatan la aplicabilidad de las teorías comportamentales en el tratamiento del dolor crónico y algunos estudios científicos que corroboran la eficacia de esta teoría para el manejo del dolor. A partir de la literatura encontrada, se concluye que a las teorías comportamentales contribuyen para el entendimiento del dolor crónico teniendo en vista que contingencias ambientales y pensamientos pueden tener un papel destacado al inicio, en la gravedad, y en el mantenimiento del dolor. Se cree, por lo tanto, que estos hallazgos pueden ser de gran valor para los profesionales contribuyendo con mejores prácticas en el ámbito de la salud.

Palabras clave: dolor crónico, conductual, terapia conductual, manejo del dolo

\footnotetext{
${ }^{1}$ Endereço de contato: Rua Fortaleza, 3180, bloco I, apto. 11 - Residencial Vila Germania. CEP 85807-090, Cascavel, PR.
} 


\section{Introdução}

De acordo com a Organização Mundial de Saúde (OMS), a dor crônica afeta 30\% da população do mundo, sendo uma das principais causas de afastamento do trabalho e aposentadoria por doença, interferindo significativamente na qualidade de vida do sujeito (OMS, 2012 apud Barros, Duarte, \& Lopes, 2014). A Associação Internacional para o Estudo da Dor (IASP- International Association for the Study of Pain) definiu a dor como "uma experiência sensorial e emocional desagradável associada a uma lesão, real ou potencial, ou descrita em termos de tal lesão" (Marquez, 2011, p. 28). A dor é essencial para a vida humana, pois, por meio dela, pode-se perceber que algo no organismo não está ocorrendo da forma esperada. No entanto, muitas vezes, a dor se torna algo recorrente e pode vir a se tornar crônica, o que atrapalha a qualidade de vida em diversos aspectos (Holtz \& Stechman, 2008).

Ao contrário da dor aguda que exerce um papel de alerta no organismo, por estar associada a lesões nos tecidos ou órgãos, a dor crônica é mais que apenas um sintoma, é a doença que persiste, não desaparecendo após a cura da lesão ou podendo ser relacionada a processos patológicos crônicos. Sua presença é constante e tem duração prolongada, podendo acarretar alterações nas mais diversas esferas da vida do sujeito, envolvendo fatores emocionais, culturais, entre outros (Sallum, Garcia, \& Sanches, 2012).

Quando torna-se crônica, a dor se transforma em um problema de saúde pública, causador de morbidade, absenteísmo ao trabalho e incapacidade temporária ou permanente, gerando altos custos aos sistemas de saúde (Picavet \& Schouten, 2003). A dor crônica leva o indivíduo a manifestar sintomas como alterações de sono, apetite, libido, irritabilidade, energia, diminuição da capacidade de concentração e restrições na capacidade para as atividades familiares, profissionais e sociais. A persistência da dor prolonga a existência desses sintomas, podendo exacerbá-los (Kreling, Cruz, \& Pimenta, 2006; Ferreira, 2001).

De acordo com Sardá Junior, Nicholas, Pimenta, \& Asghari (2012), apesar de a dor crônica interferir na capacidade funcional das pessoas, isto não é um fenômeno universal, pois alguns indivíduos parecem se ajustar bem às dores crônicas. O presente artigo trata-se de uma pesquisa bibliográfica, de caráter conceitual, realizadas por meio de banco de teses e dissertações, bibliotecas eletrônicas como Scielo, Bireme, Pepsic, livros e revistas científicas, utilizando para a pesquisa os seguintes termos: dor crônica, comportamental, cognitivo comportamental, terapia comportamental. A pesquisa teve por objetivo realizar uma análise conceitual sobre a compreensão da dor crônica na perspectiva comportamental, bem como, através da literatura, abordar técnicas e ferramentas de intervenção psicológicas utilizadas para o manejo da dor.

\section{A Compreensão Comportamental da Dor}

Diversas são as teorias de cunho fisiológico e de cunho psicológico que estudam os processos de dor. A teoria de cunho psicológico, voltada para uma abordagem comportamental compreende a dor como um comportamento, entendendo que ela não acontece ao acaso, sofre influência do meio em que o organismo vive e também o influencia (Sousa \& de-Farias, 2014).

Para B. F. Skinner, os comportamentos podem ser compreendidos enquanto ações operantes e respondentes. O comportamento operante é um comportamento voluntário e que 
opera nas contingências, sendo um comportamento que gera uma consequência, e essa consequência reforça ou enfraquece o comportamento que o gerou. Em outras palavras, o sujeito emite uma resposta que produz uma alteração no ambiente, que, por sua vez, seleciona o comportamento futuro. A ação respondente, por outro lado, é aquela em que um estímulo do ambiente elicia uma resposta específica no organismo, sendo esta uma reação fisiológica (Moreira \& Medeiros, 2009). De acordo com a teoria comportamental, a dor "sensorial" é um reflexo, sendo um respondente, enquanto que a dor "psicológica" é um ato instrumental, ou seja, um operante (Rachlin, 2010). De acordo com Skinner (1938), os eventos ambientais que definem os respondentes ocorrem antes do comportamento, enquanto que os eventos ambientais que definem os operantes ocorrem após o comportamento.

É possível saber quando alguém sente dor, pois esta é acompanhada pela emissão de um comportamento público, tal como: queixar-se da dor, gemer, fazer gestos, ou simplesmente verbalizar a ocorrência da dor. Tal atitude serve para comunicar o que está ocorrendo e pedir auxílio. No entanto "a exibição desse comportamento na ausência de dor constitui o comportamento de dor crônica" (Lobato, 2010, p. 247). Isso expressa uma resposta condicionada ou aprendida, pois o indivíduo, mesmo sem um ferimento, responde às circunstâncias sociais ou ambientais com as quais o estímulo esteve associado com frequência.

Segundo Fordyce (1976), a dor pode ser concebida tanto como um comportamento operante quanto um respondente, sendo que a dor operante desenvolve-se a partir da respondente. No princípio, a dor está correlacionada a algum estímulo antecedente eliciador da dor, como, por exemplo, um ferimento. Nesse sentido, por ser uma resposta automática e instantânea do organismo, a dor é concebida como um comportamento respondente. No entanto, se ocorrer em um ambiente que oferece reforço, como por exemplo, o acesso à medicação, redução da atividade física, atenção, preocupação de amigos e familiares, então pode se desenvolver um comportamento de dor operante, que é aprendido e se mantém ou extingue-se conforme as reações do meio.

Um exemplo é o descrito na Tabela 1, na qual se expõem os comportamentos indicativos de dor de uma criança, segundo os paradigmas respondente e operante. No que se refere ao paradigma respondente, pode-se verificar que, diante de um estímulo antecedente eliciador da dor (i.e, cair nas pedras e esfolar o joelho), a criança apresenta comportamentos reflexos de dor (sensações fisiológicas de dor e choro). Os comportamentos de dor, nesse caso, estão sob controle da condição antecedente ao comportamento. Todavia, suponha que a criança, ao chorar, recebe atenção e consolo dos pais. A consequência fornecida pelos pais pode funcionar como um reforçamento positivo e, assim, aumentar a probabilidade de a criança chorar na presença destes, em situações semelhantes no futuro. Nesse caso, pode-se dizer que o choro trata-se de um comportamento operante, uma vez que está sob controle das consequências do ambiente. Em futuras condições, pode ser que a criança caia e nem mesmo se machuque, todavia, ao observar a presença dos pais no ambiente, emita a ação de chorar, prevendo a consequência reforçadora que seguirá a tal comportamento. 
Tabela 1

Modelo de paradigma respondente e operante

\begin{tabular}{|ccc|}
\hline \multicolumn{4}{|c|}{ Paradigma Respondente } \\
\hline Antecedente & Paradigma Operante & Resposta \\
Cair das pedras e esfolar o joelho & Resposta & Consequência \\
\hline Antecedente & Sensação dolorosa e choro & Atenção dos pais $(\mathrm{R}+)$ \\
\hline $\begin{array}{c}\text { Cair na grama e se machucar } \\
\text { Cair na grama e não se } \\
\text { machucar; }\end{array}$ & Chorar & Atenção dos pais $(\mathrm{R}+)$ \\
Presença dos pais $(\mathrm{Sd})$ & & \\
\hline
\end{tabular}

Fonte: Adaptado de Burd (2010).

Os analistas entendem comportamento de dor por meio da análise funcional, sendo que esta possibilita a identificação e compreensão das contingências.

Uma formulação das interações entre um organismo e o seu meio ambiente, para ser adequada, deve sempre especificar três coisas: 1) a ocasião na qual ocorreu a resposta, 2) a própria resposta e 3) as consequências reforçadoras. As relações entre elas constituem as "contingências de reforço". (Skinner, 1975, p. 182).

A análise funcional apresentada na figura 1 teve como base comportamentos e eventos ambientais descritos por Fordyce (1976), Rachlin (2010) e Lobato (2010). Tal análise funcional objetiva realizar um esboço das possíveis contingências relacionadas ao comportamento de dor.

Segundo Chapman e Bonica (1983), como citado em Lobato (2010), várias situações podem funcionar como ocasião para o comportamento de dor: a perda de entes amados, a exposição a situações difíceis, desajustes profissionais, responsabilidades sociais pesadas e conflitos conjugais são alguns exemplos dessa natureza. O autor menciona que as exposições a eventos aversivos sejam no ambiente laboral ou familiar, ou as situações que fujam do controle da pessoa, são potenciais variáveis antecedentes a essa classe de respostas.

Na presente análise, os comportamentos avaliados são os operantes públicos da dor, ou seja, as ações de verbalizar a dor, expressar comportamentos não verbais indicativos de dor, empalidecer, corar, alterações no pulso, e outras alterações autonômicas mediadoras das indicações de dor, pedido de assistência etc. Várias são as consequências reforçadoras que podem atuar na manutenção de tais classes de respostas, assim como muitas atuam como estimulações punitivas, produzindo ainda mais sofrimento ao indivíduo com dor (Fordyce, 1976).

No que se refere às contingências de reforçamento positivo, pode-se citar a atenção social dos familiares e de outras pessoas do ambiente, a atenção de profissionais da saúde que prestam o atendimento ao sujeito, repouso, acesso à medicação, compensação financeira e, como consequências reforçadoras negativas, evitar reprovação social, obrigações ou responsabilidades indesejadas, alívio obtido pela medicação, esquiva de brigas e conflitos sociais, bem como de relações sexuais indesejadas e redução da atividade física. Portanto a dor como um operante produz consequências e é alterada por elas quando emitida em situações específicas (Fordyce, 1976; Keefe, 1982). 


\begin{tabular}{|c|c|c|}
\hline Antecedente & $\begin{array}{c}\text { Comportamentos verbais e não } \\
\text { verbais }\end{array}$ & $\begin{array}{l}\text { Consequências } \\
\text { (reforço positivo) }\end{array}$ \\
\hline $\begin{array}{l}\text {-Perda de entes amados } \\
\text {-Desajustes profissionais } \\
\text {-Sobrecarga de atividades } \\
\text { ocupacionais } \\
\text {-Responsabilidades sociais } \\
\text { aversivas } \\
\text {-Discórdia conjugal } \\
\text {-Conflitos interpessoais } \\
\text {-Estressores do cotidiano }\end{array}$ & $\begin{array}{l}\text {-Empalidecer, corar, alterações } \\
\text { no pulso, e outras alterações } \\
\text { autonômicas mediadoras das } \\
\text { indicações de dor } \\
\text {-Sinais visíveis e audíveis, } \\
\text { embora não verbais, da } \\
\text { experiência de dor (gemidos, } \\
\text { espasmos, postura } \\
\text { compensatória, movimentos } \\
\text { limitados etc.) } \\
\text {-Relatos verbais de dor } \\
\text { Pedido de assistência } \\
\text { (medicação, massagem, bolsa de } \\
\text { calor, contato com a equipe } \\
\text { etc.) }\end{array}$ & $\begin{array}{l}\text {-Evitar reprovação social } \\
\text {-Alívio obtido pela medicação } \\
\text {-Evitar obrigações ou } \\
\text { responsabilidades indesejadas } \\
\text {-Esquiva de brigas e conflitos } \\
\text { sociais } \\
\text {-Esquiva de relações sexuais } \\
\text { indesejadas } \\
\text {-Redução da atividade física }\end{array}$ \\
\hline
\end{tabular}

Figura 1. Análise funcional de um comportamento de dor Fonte: Fordyce (1976), Rachlin (2010) e Lobato (2010).

A concepção filosófica do behaviorismo radical é monista, ou seja, postula que não existe divisão entre mente e corpo. Os seres, então, têm uma única natureza que é a corpórea. Nessa concepção, dor ou sofrimento tem em comum o fato de serem privados, pois o processo sensorial da dor é uma resposta a estímulos privados, mas também produto das contingências sociais (Skinner, 1989/1991). Contudo a comunidade não tem acesso aos estímulos que controlam os comportamentos de dor, mas ela pode determinar a sua topografia de respostas (Ex.: aumento das queixas, pedido de medicação) por meio do reforçamento (Martins \& Vandenberghe, 2006).

Considera-se que a dor está sujeita aos três níveis que afetam o comportamento: filogenético, ontogenético e cultural. As características filogenéticas são as que foram selecionadas ao longo da evolução, permitindo aos indivíduos sentir dor ao terem contato com determinados condições do ambiente. Ao longo da evolução, também foram selecionadas outras 
características do organismo que permitem ao indivíduo aprender com suas experiências particulares, ou seja, sua história ontogenética (Hunziker, 2010).

Quanto à cultura, os métodos de educação de diferentes culturas influenciam as condutas e expectativas relacionadas à dor. A rigidez diante da dor em meninos é um componente cultural associado a valores como coragem e virilidade, enquanto que a expressão de dor em meninas é mais tolerada, visto que meninas podem apresentar fragilidade e vulnerabilidade na cultura ocidental (Pimenta \& Portnoi, 1999). Não apenas o sentimento, mas também a expressão da dor rege-se por códigos culturais, determinando as formas na qual ela será manifestada. Em culturas estoicas, nas quais se valoriza o autocontrole, por exemplo, a dor será vivenciada e suportada distintamente de outras culturas sem esses valores (Sarti, 2001 ).

De acordo com Souza e Laurenti (2017), é de grande importância a investigação das contingências que modelam e mantêm os comportamentos de dor, já que a modificação comportamental depende da compreensão dessas variáveis, investigando também como a dor interfere no cotidiano, quais recursos que o indivíduo utiliza para lidar com isso e o que pode estar contribuindo para a manutenção ou aumento da percepção de dor.

A teoria comportamental, em suas diferentes ondas, abrange uma série de intervenções e ferramentas que podem ser úteis para o manejo da dor. Treino de habilidades sociais, técnicas de relaxamento, desenvolvimento de repertórios deficitários, entre outros.

\section{Técnicas e Ferramentas para Manejo da Dor}

Há diversas estratégias comportamentais para o tratamento da dor crônica. As técnicas baseadas na Análise do Comportamento Aplicada, utilizando modelos propostos pelo condicionamento clássico e operante, demonstram eficácia na redução da dor em diferentes estudos encontrados na literatura (Vlaeyen et al., 2002; Fordyce, 1976). Além disso, ferramentas utilizadas na Terapia Cognitivo-Comportamental, Terapia de Aceitação e Compromisso, e Terapia Analítico-Funcional também demonstram resultados interessantes, na diminuição da intensidade da dor (Salvetti et al., 2012), identificação dos padrões comportamentais de dor e alívio (Vandenberghe, Cruz, \& Ferro, 2003), assim como na aceitação da condição dolorosa (Dahl, Wilson, Luciano, \& Hayes, 2009).

\section{O Modelo do Condicionamento Clássico e Operante}

Há diversas estratégias comportamentais para o tratamento da dor crônica, dentre elas o modelo de condicionamento clássico ou reativo citado por Turk, Burwinkle e Thieme (2011), no qual, se um estímulo doloroso for combinado repetidamente com um estímulo neutro, este último acabará por provocar a resposta à dor. Por exemplo, o indivíduo que sentiu dor depois de fazer um exercício pode ficar condicionado a experimentar uma resposta emocional negativa frente à presença do exercício e a qualquer estímulo associado ao exercício. A reação emocional negativa pode gerar tensão muscular e, desse modo, agravar a dor e reforçar ainda mais a associação entre esse estímulo e o sintoma doloroso. Com base nessa correlação condicionada, os indivíduos que padecem de dor crônica podem evitar as atividades que antes foram associadas ao desencadear ou à agravação da dor. O tratamento da dor nesse modelo consiste em estimular a exposição do indivíduo à tarefa temida, aumentando progressivamente as atividades físicas, apesar do medo da lesão e do desconforto associa- 
do. Frente à exposição, tal atividade provocará menor dor do que se previa e, com isso, há uma redução do medo antecipado e da ansiedade associados à atividade. Um estudo mais detalhado mostrou que exposição sistemática ao vivo produz reduções dramáticas no medo da dor e, com um certo atraso, também diminuições significativas na intensidade da dor e na incapacidade relacionada com dor (Vlaeyen et al., 2002).

Também há o modelo operante que se baseia na relação entre os comportamentos de dor e a sua manutenção por regras, ou pelas consequências fornecidas pelo ambiente social. $\mathrm{Na}$ análise aplicada do comportamento, a intervenção psicológica é imprescindível no sentido de identificar as variáveis contextuais que aumentam a probabilidade de comportamentos de risco ou saudáveis ocorrerem. Tal tarefa é possível por meio da análise funcional, uma vez que, ao identificar as variáveis mantenedoras do comportamento de dor, será possível modificar as contingências. O objetivo deve ser levar o paciente a desenvolver repertório comportamental alternativo para lidar melhor com sua condição (Neves \& Molina, 2015).

De acordo com Cruz (2011), as primeiras evidências em suporte do condicionamento operante na dor crônica partiram de Fordyce e colegas. No entanto os estudos existentes acerca da eficácia das técnicas de condicionamento operante são escassos e não seguem, muitas vezes, o protótipo preconizado por Fordyce.

\section{Terapia Cognitivo-Comportamental e Contribuições para o Manejo da Dor}

A Terapia Cognitivo-Comportamental (TCC) tem sido recomendada, em âmbito internacional, para o tratamento da dor (Ehde, Dillworth, \& Turner, 2014). No modelo cognitivo o que se destaca é o papel causal das crenças e distorções cognitivas, que podem intensificar a percepção da dor. Nesse modelo, o objetivo é mudar os pensamentos e crenças. A prioridade em seu tratamento é ensinar o cliente a enfrentar e reduzir a dor através de treino de relaxamento, distração cognitiva e visualização e, num segundo momento, ensiná-lo a utilizar as técnicas cognitivas para rebater as crenças, pois, de acordo com a terapia cognitivo comportamental (TCC), o pensamento é um dos fatores que influenciam a dor, podendo até mesmo agravar sua intensidade (Angelotti, 2001).

Em um estudo feito por Salvetti et al. (2012), pacientes com dor crônica foram avaliados antes e ao final de um programa no qual se utilizaram estratégias cognitivo-comportamentais. Ao final do programa, observou-se redução significativa na intensidade da dor, de incapacidade relacionada à dor e de sintomas depressivos. Uma outra pesquisa que concluiu a efetividade da terapia cognitivo-comportamental no tratamento da dor crônica foi realizada por Morley, Eccleston e Williams (1999) que analisou estudos randomizados que utilizaram a TCC, no tratamento da dor crônica, concluindo que tratamentos baseados nessa abordagem produziram mudanças significativas na dor, no humor, nas estratégias de coping, no comportamento doloroso, no nível de atividade e no desempenho social dos sujeitos pesquisados.

As revisões da literatura (Keefe, Dunsmore, \& Brunett, 1992; Turk \& Okifuji, 2002) demonstram que, quando a eficácia dos métodos da Análise Aplicada no tratamento da dor crônica é comparada com a da Terapia Comportamental Cognitiva, em pesquisa de cunho hipotético-dedutiva com delineamento de grupos, os resultados são muito similares, inclusive na mudança de variáveis cognitivas. Verifica-se que, apesar de focalizar somente frequências de comportamentos públicos, os métodos operantes também afetam crenças e pensamen- 
tos do sujeito (Vandenberghe, 2005).

As técnicas de relaxamento são muito utilizadas no manejo da dor crônica, sendo eficazes porque ensinam os pacientes a produzir uma resposta que reduz o estresse e a dor (Salvador, Rodrigues, \& Carvalho, 2008). O relaxamento pode ser definido com um esforço geral para se diminuir a excitabilidade do organismo. Podem ser usadas diferentes técnicas com esse objetivo, como a respiração diafragmática, imagem mental relaxante e relaxamento muscular progressivo.

A respiração diafragmática se constitui em uma técnica de relaxamento que visa à diminuição da ansiedade. Nessa técnica, pede-se que o indivíduo preste atenção em sua respiração e identifique os movimentos de inspirar e expirar colocando a mão sobre o abdômen e a região peitoral. Em seguida, pede-se que respire lenta e pausadamente, inspirando por três segundos, segurando a respiração por mais três segundos e soltando a respiração pela boca por seis segundos. Essa respiração impede a hiperventilação e diminui os sintomas autonômicos e a tensão muscular (Oliveira \& Duarte, 2004). Pode-se aliar a respiração diafragmática a uma imagem mental relaxante. Nesse caso, pede-se ao paciente para deixar vir à mente uma imagem que lhe cause tranquilidade. Em seguida, o terapeuta explora os detalhes mais relaxantes e as sensações. A descrição da cena deve incluir os elementos emocionais como sinais visuais, sons, sensações de movimento e temperatura, que induzem a uma sensação de tranquilidade (Penido \& Pereira, 2010).

Já a distração cognitiva consiste na mudança do foco de atenção para outras situações que podem ser agradáveis e, muitas vezes, encontram-se disponíveis no próprio ambiente. A influência da atenção na amplificação da dor é um processo bastante estudado. Diversos autores concordam que a atenção dirigida para dor aumenta a intensidade da experiência dolorosa e que distrair a atenção da dor diminui a intensidade da experiência dolorosa (Keefe et al., 1992; Pincus \& Morley, 2001; Borges, Luiz, \& Domingos, 2009).

O relaxamento muscular progressivo proposto por Jacobson ajuda o paciente a identificar diferentes níveis de tensão do organismo e livrar os músculos dessa tensão (Rangé, 2001). Nessa técnica, é utilizada a contração muscular seguida de relaxamento, de diversos grupos musculares, iniciando pelos membros, depois o tronco e a cabeça. Através desse treino, a pessoa aprende a identificar e diferenciar níveis de tensão muscular podendo relaxar a musculatura quando ainda está se iniciando a tensão (Penido \& Pereira, 2010).

Um estudo feito por Paula, Carvalho, e Santos (2002) com o objetivo de verificar o nível de dor em pacientes pós-cirúrgicos, antes e após a aplicação da técnica de Relaxamento Muscular Progressivo, concluiu que o uso desta permitiu aos sujeitos avaliar que seu nível de dor diminuiu. Quando o relaxamento muscular progressivo é praticado e utilizado no dia a dia, ele pode ajudar a neutralizar alguns dos efeitos da reação ao estresse, como o aumento da tensão muscular.

\section{Terapias de Terceira Onda e as Contribuições no Contexto da Dor}

Nas Terapias de Terceira Onda, a ênfase está no contexto e na função do comportamento, e não na sua forma e conteúdo. Os principais modelos desenvolvidos pela terceira geração e que iremos desenvolver neste trabalho são: a Terapia Comportamental-Dialética (Dimeff \& Linehan, 2001), Terapia de Aceitação e Compromisso-ACT (Hayes, Strosahl, \& Wilson, 2012), 
e Psicoterapia Analítica-Funcional-FAP (Kohlenberg \& Tsai, 2001).

Essas terapias estão comprometidas com a experiência direta acima da compreensão racional, e com a promoção de aceitação de eventos privados aversivos, o oposto do controle, e enfatizam as trocas espontâneas entre terapeuta e cliente como elementos chave do processo terapêutico (Vandenberghe, 2002, 2007). As terapias de Terceira Onda têm alcançado resultados promissores no tratamento de diversos problemas de comportamento e transtornos psiquiátricos, internacionalmente (Callaghan et al., 2012; Cattivelli, Tirelli, Berardo, \& Perin, 2012) e no Brasil (Oshiro, Kanter, \& Meyer, 2012; Pezzato, Brandão, \& Oshiro, 2012).

A utilização do Mindfulness, como tratamento para dor crônica, relacionado com as práticas de terceira onda das Terapias Comportamentais, refere-se um estado mental ou psicológico caracterizado pela regulação intencional da atenção ao que está acontecendo no exato momento, ou seja, quando o sujeito conduz sua atenção de forma sustentada a cada atividade ou fenômeno enquanto este está ocorrendo. O Mindfulness apresenta características específicas de registrar as emoções, pensamentos e comportamentos no momento presente: apenas observar os fatos e situações sem avaliar, analisar ou julgar, focar no presente, sem ligar-se ao passado ou futuro (Kabat-Zinn, 2007). Pode-se dizer, de forma breve, que Mindfulness é a habilidade de estar consciente dos seus pensamentos, emoções, sensações e ações, no momento presente sem julgar ou criticar a si mesmo ou à própria experiência. Com a prática é possível que o indivíduo portador de dor crônica promova uma perspectiva flexível de sua realidade, promovendo melhor adesão ao tratamento proposto, aumentando, dessa forma, a habilidade de adaptação, fortalecendo a aceitação e consequentemente redução da dor. As estratégias e técnicas de Mindfulness são utilizadas em terapias como a ACT e FAP (Vandenberghe \& Assunção, 2009).

A Terapia de aceitação e compromisso também traz contribuições importantes para o tratamento da dor crônica, e é um dos tratamentos psicológicos que têm maior apoio empírico na abordagem da dor (Hayes, Strosahl, \& Wilson, 2012). O que a ACT propõe é que o cliente não evite pensar na dor, pois obterá como consequência ser lembrado da dor, ou seja, a fuga da dor, na verdade, cria mais dor. Ao tentar controlar a dor, muitas vezes as pessoas abandonam o trabalho, vida social e tudo que poderia melhorar a sua vida. Esses comportamentos de esquiva tendem a aumentar a probabilidade de novas respostas aversivas e dolorosas (Martins \& Vandenberghe, 2006). Em vez de aliviar ou controlar o "problema", os clientes aprendem a aceitar experiências particulares e a buscar objetivos mais significativos a longo prazo (Dahl et al., 2009).

Em um estudo de McCracken \& Samuel (2007), foi investigado o padrão de atividade de 276 indivíduos que apresentavam dor crônica. Esse estudo demonstrou que clientes que evitaram atividades sofreram maior incapacidade e desgaste físico do que aqueles que não a evitaram. A intensidade ou a severidade da dor não distingue grupos, mas sim a aceitação da dor. Nesse estudo, os grupos com maior fuga e incapacidade relataram menores graus de aceitação da dor.

Muitas vezes indivíduos com dores crônicas evitam contatos sociais, podendo resultar em isolamento, e em muitos casos levar à depressão. Portanto a estimulação para que o paciente se engaje em atividades sociais reforçadoras, mantenha sua rede de relações e não se isole quando estiver com dor é uma questão importante a ser trabalhada (Sardá Junior, 2015). A esquiva emocional nesses casos impede que o sujeito entre em contato com as 
contingências reais. No contexto da dor, a experiência dolorosa é reforçada quando a pessoa abandona atividades como o trabalho, vida social e atitudes que poderiam melhorar sua qualidade de vida, tornando os comportamentos de dor cada vez mais frequentes. Esses comportamentos associados a estratégias de esquiva aumentam a probabilidade de novas respostas dolorosas, estabelecendo uma fonte de manutenção da dor. Nesse círculo vicioso, a dor se torna uma estratégia para solução de problemas, utilizando dele para substituir a tomada de decisão assertiva (Martins \& Vandenberghe, 2007). A utilização dessa estratégia para lidar com estímulos dolorosos intensos aumenta consideravelmente os níveis de estresse e a magnitude da dor (Queiroz, 2009).

A dor é um sentimento inerente à vida, ou seja, ela pode ser minimizada, mas não excluída. Ela pode ser benéfica, necessária à sobrevivência, mas pode também se tornar um problema. O enfrentamento dessas contingências, que pode depender de haver ou não alternativas vigentes, vai determinar a qualidade de vida dos indivíduos a elas submetidos (Hunziker, 2010). A ACT nesse sentido não irá ajudar a evitar a dor. O que ela propõe é uma mudança na maneira de gerir as experiências pessoais. A proposta da ACT é, então, abandonar as tentativas de controlar a dor, o que implica a necessidade de reconstruir novos contextos sócio-verbais, reconhecendo-a e aceitando-a como algo que faz parte de sua vida, descobrindo que viver com dor não implica incapacidade, que vale a pena viver mesmo com ela (Sousa \& de-Farias, 2014).

Além da ACT, Vandenberghe, Cruz, e Ferro (2003) citam que a Psicoterapia Analítico Funcional (FAP), também traz grandes contribuições em casos de dor crônica. Na FAP o objeto de análise se dá no relacionamento terapeuta-cliente. Nela se considera o contexto privilegiado em que os comportamentos ocorrem como uma oportunidade única de interação que poderá permitir mudanças nos comportamentos. A recusa do terapeuta em reforçar o comportamento de fuga e esquiva problemático e o cuidado de identificar e reforçar pequenas mudanças constitui a possibilidade de modelar repertórios enquanto eles estão realmente acontecendo.

Vandenberghe, Cruz e Ferro (2003) descrevem intervenções em grupo com portadores de dor crônica utilizando a FAP, com o objetivo de ajudar o indivíduo a identificar os comportamentos diante dos episódios de dor e que recursos normalmente são utilizados para aliviar a sua dor. O terapeuta também pode examinar as funções das queixas e sintomas no ambiente social, com o objetivo de quebrar o círculo vicioso da dor, modificando a maneira como o cliente compreende e interage com a dor e possibilitando um contato maior com as contingências. No grupo as relações que se desenvolvem são o contexto no qual mudanças podem ocorrer, tendo a possibilidade de discussão sobre o significado da dor para os diferentes integrantes, aumentando assim as possibilidades terapêuticas, e oportunizando trocas entre eles. Os participantes agem em uma situação real, na qual consequências naturais estão acessíveis. A FAP, nesse sentido, enfatiza as relações estabelecidas como oportunidade para modelagem e modelação de comportamentos, por meio do manejo das contingências realizadas pela terapeuta (Neves \& Molina, 2015).

De acordo com Vandenberghe et al. (2003), nesse estudo em grupo utilizando a FAP, os comportamentos clinicamente relevantes (CCR1) que mantêm a dor crônica podem ocorrer no grupo, e são enfraquecidos pelo terapeuta, assim como os progressos ao vivo (CCR2), 
são reforçados, e as análises realizadas pelo paciente (CCR3) são modeladas. Para isso, o terapeuta precisa observar cuidadosamente os efeitos potencialmente reforçadores que as suas reações têm sobre os comportamentos do paciente e compartilhar com ele as suas interpretações das variáveis que afetam o seu comportamento.

\section{Contribuições do Treinamento de Habilidades Sociais para o Tratamento da Dor}

Na literatura estudos têm incluído o treino assertivo e de habilidades de comunicação no tratamento de pacientes com dor crônica (Winterowd, Beck, \& Gruener, 2003; Penido, Rangé, \& Fortes, 2005). Um estudo realizado por Penido (2004), sobre habilidades sociais em pacientes com dor, mostrou que, em comparação com um grupo controle sem dor crônica, os grupos com dor apresentaram um repertório de habilidades sociais mais comprometido, como maior dificuldade nas habilidades assertivas de dizer não e pedir mudança de comportamento.

O comportamento de dor pode ocorrer por um processo de reforçamento, podendo se dar diretamente pela atenção da família e pela atenção médica, ou pela esquiva de situações desagradáveis ou obrigações penosas. O fato de estar com dor pode funcionar para evitar determinadas tarefas desagradáveis, como arrumar a casa ou dizer não. Nesse caso, a falta de algumas habilidades sociais de enfrentamento, como assertividade, poderia funcionar como um fator que aumenta a probabilidade dos comportamentos de dor ocorrer (Fordyce et al., 1973). Para Winterowd, Beck, e Gruener (2003), o treinamento de habilidades sociais para pacientes com dor crônica deve incluir aprender a prestar atenção e ouvir o outro; aprender a resumir o que ouviu do outro e devolver, sem julgamentos ou críticas, procurando ser objetivo e específico nessa comunicação; aprender a comunicar a própria opinião, sentimentos, necessidades e vontades usando frases iniciadas pelo pronome eu; aprender a colocar em prática esses comportamentos buscando resolver situações e melhorar as relações. Para tal é preciso ter consciência dos próprios direitos e direitos dos outros, além de buscar identificar o momento adequado para a interação.

\section{Conclusão}

O objetivo geral desta pesquisa foi o de realizar uma análise conceitual sobre o entendimento da dor crônica, sob a perspectiva comportamental e intervenções psicológicas que pudessem ser utilizadas no manejo clínico desses quadros. Conclui-se, conforme os estudos apontados, que indivíduos com dor crônica podem se beneficiar de intervenções psicológicas embasadas nessa teoria, pois possibilitam aprender a lidar de uma forma diferente com a experiência dolorosa, diminuindo o seu sofrimento, aumentando sua resiliência e melhorando sua qualidade de vida. As ferramentas utilizadas pela Terapia de Aceitação e Compromisso, os procedimentos clínicos adotados pela Psicoterapia Analítico Funcional, as técnicas de exposição gradual, as técnicas de relaxamento, o treinamento assertivo e de habilidades sociais são algumas das ferramentas que mostram eficácia no manejo do quadro de dor. A análise funcional do comportamento de dor utilizada pela teoria comportamental também fornece uma compreensão das variáveis envolvidas na manutenção do quadro doloroso.

É de suma importância pesquisar a amplitude de fatores que contribuem para a experi- 
ência da dor, e as influências que ela exerce na vida do indivíduo e das pessoas às quais se relaciona. Devem-se entrelaçar diversos saberes ampliando o foco de atenção e cuidados a esses sujeitos, tendo em vista que a dor crônica causa sofrimento em diversas áreas da vida.

Como exposto no decorrer deste estudo, é evidente que há uma série de recursos, técnicas e estratégias cognitivas e comportamentais que podem ser utilizadas no tratamento de pessoas com dor crônica, visando ajudá-las a adaptarem-se ao diagnóstico e conviverem meIhor com as implicações físicas e psicológicas decorrentes da nova condição, proporcionando ao sujeito uma vida mais funcional independente de sua condição.

Como principal limitação da presente pesquisa, deve-se destacar que os resultados obtidos são parte de análises fundamentalmente teóricas da literatura existente sobre os temas abordados. Sugere-se, portanto, a necessidade de se realizar mais estudos e pesquisas na área que contemplem métodos experimentais e aplicados, e em diferentes tipos de população com dor crônica, com o objetivo de aprofundar as ferramentas de avaliação e intervenção do quadro de dor.

\section{Referências}

Angelotti, G. (2001). Tratamento da dor crônica. In B. Rangé (Org.), Psicoterapias cognitivocomportamentais: Um diálogo com a psiquiatria (pp. 535-545). Porto Alegre: Artmed.

Barros, J. R. F., Duarte, M. G. O., \& Lopes, A. P. (2014). A terapia cognitivo-comportamental no tratamento de pacientes com dor crônica. Caderno de Graduação-Ciências Biológicas e da Saúde, 2(2), 77-90. Disponível em https://periodicos.set.edu.br/index.php/fitsbiosaude/ article/view/1536/1045

Borges, C. S., Luiz, A. M. A. G., \& Domingos, N. A. M. (2009). Intervenção cognitivocomportamental em estresse e dor crônica. Arquivos de Ciências da Saúde, 16(4), 181-6.

Burd, J. M. F. M. (2010). Psicossomática hoje. In O. Lobato. O problema da dor (pp.235-254). Porto Alegre: Artmed, 2010.

Callaghan, G. M., Duenas, J. A., Nadeau, S. E., Darrow, S. M., Merwe, J. V., \& Misko, J. (2012). An empirical model of body image disturbance using behavioral principles found in functional analytic psychotherapy, acceptance and commitment therapy. International Journal of Behavioral Consultation Therapy, 7(2), 16-24.

Cattivelli, R., Tirelli, V., Berardo, F., \& Perin, S. (2012). Promoting appropriate behavior in daily life contexts using functional analytic psychotherapy in early-adolescent children. International Journal of Behavioral Consultation Therapy, 7(2), 25-32

Chapman, C. R; Bonica, J. J. Acupe pain, current concepts. New York: Upjohn, 1983.

Cruz, C. R. R. (2011). Dor crónica: Uma perspectiva biopsicossocial (Dissertação de Mestrado, Faculdade de Medicina, Universidade de Coimbra). Disponível em https:// estudogeral.sib.uc.pt/bitstream/10316/47960/1/DOR\%20CR\%C3\%93NICA\%20UMA\%20 PERSPECTIVA\%2OBIOPSICOSSOCIAL.pdf

Dahl, J., Wilson, K. G., Luciano, C., \& Hayes, S. C. (2009). Mecanismos psicocomportamentais em dor. In O. Alves Neto, C. M. C. Costa, J. T. T. Siqueira, M. J. Teixeira (Orgs.), Dor: Princípios e prática (Cap. 18, pp. 247-265). Porto Alegre: Artmed.

Dimeff, L., \& Linehan, M. M. (2001). Dialectical behavior therapy in a nutshell. The California Psychologist, 34(3), 10-13. 
Ehde, D. M., Dillworth, T. M., \& Turner, J. A. (2014). Cognitive-behavioral therapy for individuals with chronic pain: efficacy, innovations, and directions for research. American Psychologist, 69(2), 153. doi: 10.1037/a0035747

Ferreira P. E. M. S. Dor crônica: Avaliação e tratamento psicológico. In A. C. Andrade Filho (Ed.), Dor: Diagnóstico e tratamento (pp. 43-52). São Paulo: Roca, 2001.

Fordyce, W. E. (1976). Behavioral methods for chronic pain and illness. [S.I.]: C. V. Mosby.

Fordyce, W. E.; Fowler, R.; Lehmann, J.; Delateur, B.; Sand, P. \& Trieschmann, R. (1973). Operant conditioning in the treatment of chronic pain. Archives of Physical Medicine and Rheabilitation, 54, 399-408

Hayes, S. C., Strosahl, K. D., \& Wilson, K. G. (2012). Acceptance and commitment therapy. New York, NY: Guilford Press.

Holtz, V. V., \& Stechman Neto, J. (2008). Epidemiologia da dor em pacientes de Curitiba e região metropolitana. Revista Dor, 9(2), 1217-24.

Hunziker, M. H. L. (2010). Comportamento de dor: Análise funcional e alguns dados experimentais. Temas em Psicologia, 18(2), 327-333. Disponível em: http://pepsic. bvsalud.org/pdf/tp/v18n2/v18n2a07.pdf

Kabat-Zinn, J. (2007). La pratica del atención plena. Barcelona: Kairós.

Keefe, F. J. (1982). Behavioral assessment and treatment of chronic pain: Current status and future directions. Journal of Consulting and Clinical Psychology, 50(6), 896-911. doi: 10.1037//0022-006X.50.6.896

Keefe, F. J., Dunsmore, J., \& Burnett, R. (1992). Behavioral and cognitivebehavioral approaches to chronic pain: recent advances and future directions. Journal of Consulting and Clinical Psychology, 60(4), 528-536

Kohlenberg, R.J, \& Tsai, M. (2001). Psicoterapia Analítica Funcional: Criando relações terapêuticas intensas e curativas (R. R. Kerbauy, trad.). Santo André, SP: ESETec. (Publicado originalmente em 1991).

Kreling, M. C. G. D., Cruz, D. A. L. M., \& Pimenta, C. A. (2006). Prevalência de dor crônica em adultos. Revista Brasileira de Enfermagem, 59(4), 509-13.

Lobato, O. (2010). O problema da dor. In J. M. F. M. Burd, Psicossomática hoje (Cap. 17, pp. 235-254). Porto Alegre: Artmed.

Marquez, J. O. (2011). A dor e os seus aspectos multidimensionais. Ciência e Cultura, 63(2), 28-32. doi: 10.21800/S0009-67252011000200010

Martins, M. A., \& Vandenberghe, L. (2006). Psicoterapia no tratamento da fibromialgia: Mesclando FAP e ACT. In H. J. Guilhardi \& N. C. Aguirre (Orgs.), Sobre comportamento e cognição (Vol. 18: Expondo a variabilidade, pp. 238-248). Santo André, SP: ESETec.

Martins, M. A., \& Vandenberghe, L. (2007). Intervenção psicológica em portadores de fibromialgia. Revista Dor: Pesquisa, Clínica e Terapêutica, 8(4), 1103-1112.

McCracken, L. M., \& Samuel, V. M. (2007). The role of avoidance, pacing, and other activity patterns in chronic pain. Pain, 130(1), 119-125. doi: 10.1016/j.pain.2006.11.016

Moreira, M. B., \& de Medeiros, C. A. (2009). Princípios básicos de análise do comportamento. Porto Alegre: Artmed.

Morley, S., Eccleston, C., \& Williams, A. (1999). Systematic review and meta-analysis of randomized controlled trials of cognitive behaviour therapy and behaviour therapy for chronic pain in adults, excluding headache. Pain, 80(1-2), 1-13. 
Neves, M. S., \& Molina, R. A. (2015). Terapia analítico comportamental e cognitivocomportamental em grupo: Intervenções com portadores de doença crônica. UNIciências, 15(1), 129-140. Disponível em http://pgsskroton.com.br/seer/index.php/uniciencias/ article/viewFile/602/571

Oliveira, M. A., \& Duarte, A. M. M. (2004). Controle de respostas de ansiedade em universitários em situações de exposições orais. Revista Brasileira de Terapia Comportamental e Cognitiva, 6(2), 183-199.

Oshiro, C. K. B., Kanter, J., \& Meyer, S. B. (2012). A single-case experimental demonstration of functional analytic psychotherapy with two clients with severe interpersonal problems. International Journal of Behavioral Consultation Therapy, 7(2), 111-116.

Paula, A. A. D. D., Carvalho, E. C. D., \& Santos, C. B. D. (2002). The use of the" progressive muscle relaxation" technique for pain relief in gynecology and obstetrics. Revista LatinoAmericana de Enfermagem, 10(5), 654-659. doi: 10.1590/S0104-11692002000500005

Penido, M. A (2004). A influência das habilidades sociais em pacientes fibromiálgicas (Dissertação de mestrado, Instituto de Psicologia, Universidade Federal do Rio de Janeiro).

Penido, M. A., Rangé, B., \& Fortes, S. (2005). Um estudo investigando as habilidades sociais de pacientes fibromiálgicas. Revista Brasileira de Terapias Cognitivas, 1(2), 75-86.

Penido, M. A., \& Pereira, F. M. (2010). Aplicabilidade teórico-prática da terapia cognitivo comportamental na psicologia hospitalar. Revista Brasileira de Terapias Cognitivas, 6(2), 189-220. doi: https://dx.doi.org/10.5935/1808-5687.20100021

Pezzato, F. A., Brandão, A. S., \& Oshiro, C. K. B. (2012). Intervenção baseada na Psicoterapia Analítica Funcional em um caso de transtorno de pânico com agorafobia. Revista Brasileira de Terapia Comportamental e Cognitiva, 15(1), 74-84.

Picavet, H. S. J., \& Schouten, J. S. A. G. (2003). Musculoskeletal pain in the Netherlands: Prevalences, consequences and risk groups, the DMC3-study. Pain, 102(1-2), 167-178.

Pimenta, C. D. M., \& Portnoi, A. G. (1999). Dor e cultura. In M. M. M. J. Carvalho (Org.), Dor: Um estudo multidisciplinar (pp. 159-73). São Paulo: Summus.

Pincus, T., \& Morley, S. (2001). Cognitive-processing bias in chronic pain: a review and integration. Psychological Bulletin, 127(5), 599-617.

Queiroz, M. A. M. (2009). Psicoterapia comportamental e fibromialgia: Alvos para intervenção psicológica. Santo André, SP: ESETec.

Rachlin, H. (2010). Dor e comportamento. Temas em Psicologia, 18(2), 429-447. Disponível em http://pepsic.bvsalud.org/pdf/tp/v18n2/v18n2a17.pdf

Rangé, B. (2001). Psicoterapias cognitivo-comportamentais: Um diálogo com a psiquiatria. Rio de Janeiro: Artmed

Sallum, A. M. C., Garcia, D. M., \& Sanches, M. (2012). Dor aguda e crônica: Revisão narrativa da literatura. Acta Paulista de Enfermagem, 25(1), p. 150-154. Disponível em: http:// www.scielo.br/pdf/ape/v25nspe1/pt_23.pdf

Salvador, M., Rodrigues, C. C., \& Carvalho, E. C. (2008). Emprego do relaxamento para alívio da dor em oncologia. Revista da Rede de Enfermagem do Nordeste, 9(1). Disponível em: http://www.redalyc.org/pdf/3240/324027961015.pdf

Salvetti, M. G., Cobelo, A., Vernalha, P. M., Vianna, C. I. A., Canarezi, L. C. C. C. C., \& Calegare, R. G. L. (2012). Efeitos de um programa psicoeducativo no controle da dor crônica. 
Revista Latino-Americana de Enfermagem, 20(5), 896-902. https://dx.doi.org/10.1590/ S0104-11692012000500011

Sardá Júnior, J. J., Nicholas, M. K., Pimenta, C. A. D. M., \& Asghari, A. (2012). Preditores biopsicossociais de dor, incapacidade e depressão em pacientes brasileiros com dor crônica. Revista Dor, 13(2), 111-118. Disponível em http://www.scielo.br/pdf/rdor/ v13n2/03.pdf

Sardá Júnior, J. J. (2015). Avaliação psicológica do paciente com dor. In F. P. Minson, M. C. Morete, M. A.; Marangoni, Manuais de especialização-Dor (1a ed., Cap. 7, pp. 127-146). São Paulo: Manole.

Sarti, C. A. (2001). A dor, o indivíduo e a cultura. Saúde e Sociedade, 10(1), 3-13. doi: https:// dx.doi.org/10.1590/S0104-12902001000100002

Skinner, B. F. (1938). The behavior of organisms. New York: Appleton-Century-Crofts.

Skinner, B. F. (1975). Contingências de reforço: Uma análise teórica (Coleção Os Pensadores, vol. 51, R. Moreno, Trad.). São Paulo: Abril Cultural.

Skinner, B. F. (1989/1991). Questões recentes na análise comportamental (M. da P. Villalobos, trad.). Campinas, SP: Papirus.

Sousa, D. D. D., \& de-Farias, A. K. C. (2014). Dor crônica e Terapia de Aceitação e Compromisso: Um caso clínico. Revista Brasileira de Terapia Comportamental e Cognitiva, 16(2), 125147. Disponível em http://www.usp.br/rbtcc/index.php/RBTCC/article/viewFile/696/416

Souza, B. D., \& Laurenti, C. (2017). Uma interpretação molar da dor crônica na fibromialgia. Psicologia: Ciência e Profissão, 37(2), 363-377. doi: 10.1590/1982-3703001102016.

Turk, D., \& Okifuji, A. (2002). Psychological factors in chronic pain: Evolution and revolution. Journal of Consulting \& Clinical Psychology, 70(3), 678-690

Turk, D. C., Burwinkle, T., \& Thieme, K. (2011). Intervenções psicológicas. In J. H. V. Roenn, J. A. Paice, \& M. E. Preodor. Current: Diagnostico e tratamento da dor (Cap 05, pp. 50-62). Porto Alegre: AMGH.

Vandenberghe, L. (2002). A prática e as implicações da análise funcional. Revista Brasileira de Terapia Comportamental e Cognitiva, 4(1), 35-45.

Vandenberghe, L. (2007). Terapia comportamental construtiva: Uma outra face da clínica comportamental. Psicologia USP, 18(4), 89-102

Vandenberghe, L., \& Assunção, A. B. (2009). Concepções de mindfulness em Langer e KabatZinn: Um encontro da ciência Ocidental com a espiritualidade Oriental. Contextos Clínicos, 2(2), 124-135.

Vandenberghe, L., Cruz, A. C. F. D., \& Ferro, C. L. B. (2003). Terapia de grupo para pacientes com dor crônica orofacial. Revista Brasileira de Terapia Comportamental e Cognitiva, 5(1), 3140. Disponível em http://pepsic.bvsalud.org/scielo. php?script=sci_arttext\&pid=S1517-55452003000100005

Vlaeyen, J., de Jong, J., Onghena, P., Kerckhoffs-Hanssen, M. \& Kole-Snijders, A. (2002). Can pain-related fear be reduced? The application of exposure in vivo. Pain Research and Management, 7(3), 144-153.

Winterowd, C., Beck, A. T., \& Gruener, D. (2003). Cognitive therapy with chronic pain patients. New York: Springer Publishing Company. 
Recebido: 08/11/2017

Última revisão: $27 / 06 / 2018$

Aceite final: 02/09/2018

\section{Sobre os autores:}

Cristiane Rodrigues Lopes- Graduada em Psicologia pela Universidade Paranaense (UNIPAR). E-mail: cris_rodriguees@hotmail.com, Orcid: http://orcid.org/0000-0003-3746-1540

Vanessa Ferrari-Graduanda em Psicologia pela Universidade Paranaense (UNIPAR) E-mail: vanessa-ferrari1@hotmail.com, Orcid: http://orcid.org/0000-0003-2320-7349

Cynthia Carvalho Jorge - Mestre em Psicologia pela Universidade Federal de São Carlos (UFSCar). Especialista em Terapia Analítico-Comportamental pelo Instituto de Terapia por Contingências de Reforçamento (ITCR - Campinas). Docente da Graduação em Psicologia e da Pós-Graduação em Clínica Analítico-Comportamental da Universidade Paranaense. E-mail: cynthia@prof.unipar.br, Orcid: http://orcid.org/0000-0002-2290-388X 